\title{
Photon-sparse microscopy: visible light imaging using infrared illumination : supplementary material
}

\author{
Reuben S. Aspden, ${ }^{1}$ Nathan R. Gemmell, ${ }^{2}$ Peter A. Morris, ${ }^{1}$ \\ Daniel S. TAsCa, ${ }^{1 \dagger}$ Lena Mertens, ${ }^{1}$ Michael G. Tanner, \\ Robert A. KIRKWOOd, ${ }^{3}$ Alessandro Ruggeri, ${ }^{4}$ Alberto Tosi, ${ }^{4}$ \\ ROBERT W. BOYd, ${ }^{5,6}$ GeRALD S. BULler, ${ }^{2}$ ROBERT H. HADFIELD, ${ }^{3}$ \\ AND MILES J. PADGETT ${ }^{1, *}$ \\ ${ }^{1}$ School of Physics and Astronomy, University of Glasgow, G12 8QQ, UK \\ ${ }^{2}$ School of Engineering \& Physical Sciences, Heriot-Watt University, EH14 4AS, UK \\ ${ }^{3}$ School of Engineering, University of Glasgow, G12 8QQ, UK \\ ${ }^{4}$ Dipartimento di Elettronica, Informazione e Bioingegneria Politecnico di Milano, Piazza Leonardo da Vinci 32, 20133 Milano, Italy \\ ${ }^{5}$ Department of Physics, University of Ottawa, Ottawa, Ontario, Canada \\ ${ }^{6}$ The Institute of Optics and Department of Physics and Astronomy, University of Rochester, Rochester, NY 14627, USA \\ + Current address: Instituto de Física, Universidade Federal do Rio de Janeiro, Caixa-Postal 68528, Rio de Janeiro, RJ 21941-972, Brazil \\ ¥ Current address: School of Engineering \& Physical Sciences, Heriot-Watt University, EH14 4AS, UK \\ *Corresponding author: miles.padgett@glasgow.ac.uk
}

Published 10 December 2015

This document provides supplementary information to "Photon-sparse microscopy: visible light imaging using infrared illumination," http://dx.doi.org/10.1364/optica.2.001049. We describe the optical configuration of the full imaging system and measure the resolution of the acquired images. The theoretical resolution of the imaging system is calculated, and we discuss the main experimental factors affecting the resolution of the imaging system. We present a method of photon counting within each frame of the acquired image, and use the statistical properties of the images to reconstruct images using far fewer photons than required to develop a conventional image. (c) 2015 Optical Society of America

http://dx.doi.org/10.1364/optica.2.001049.s001

Optical configuration of imaging system

The full schematic of our experiment is shown in Figure S1. Our down-conversion source consists a pulsed (100 MHz repetition rate, 10 ps pulse duration) solid-state pump laser operating at 355 $\mathrm{nm}$ with an average output power of $100 \mathrm{~mW}$. The beam from this laser is collimated to a size of $470 \mu \mathrm{m}$ FWHM and is then incident upon a $1 \mathrm{~mm}$ long ß-barium borate (BBO) crystal. The crystal is cut for type-I phase matching such that the beams of down-converted signal and idler photons are at $460 \mathrm{~nm}$ and $1550 \mathrm{~nm}$, respectively. These photons are separated from each other using a dichroic beam splitter into two spatially distinct free-space optical paths where they are selected using high transmission interference filters. The idler path reimages the plane of the crystal to the object plane with a magnification of $M=-1 / 2$. Any photon transmitted by the object is reimaged to the plane of the SPAD with a further magnification of $M=-10$. The detection area of the SPAD is circular with a diameter of $25 \mu \mathrm{m}$, setting the width of the field of view of our imaging system to $250 \mu \mathrm{m}$. In the signal path, the plane of the crystal is reimaged to a time-gated, intensified CCD camera (ICCD), with a magnification of $M=5$. Thus the effective magnification through both arms of the imaging system from object to ICCD is $M=-10$.

The detection of an infrared photon is used to trigger the gate of the intensified camera such that the blue photon imaged on the camera and the infrared photon detected at the InGaAs/InP SPAD are from the same signal and idler photon pair. The combined response time from the input to the SPAD to the triggering of the intensifier in the ICCD (including the SPAD and the ICCD trigger 
mechanism) is approximately 75 ns. Therefore, to ensure synchronization between signal and idler photons the signal arm is extended in length using a double-pass, image-preserving delay line comprising lenses and fold mirrors. The delay line is $25.2 \mathrm{~m}$ in length. We use a polarizing beam splitter (PBS) to allow entry into the double-pass section of the delay line and rotate the polarization through $90^{\circ}$ within the delay line in order to reflect at the PBS on the return leg. The photon is then reimaged through a further telescopic imaging system before being detected by the camera.

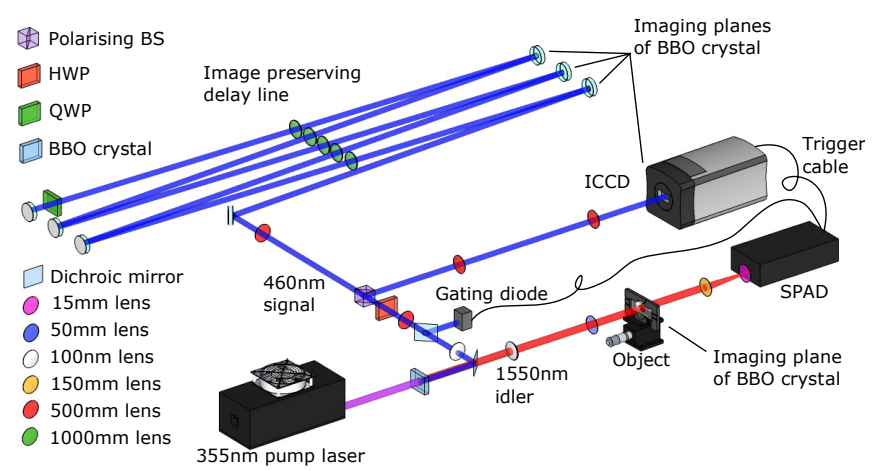

Figure S1. Full schematic of the experimental setup. The plane of the crystal is reimaged through the delay line to the ICCD camera in the signal path, and to the object plane in the idler path.

\section{Measured Resolution}

We determined the point spread function (PSF) of our system by analyzing the transition between the gold background and silicon central square in the image of the $50 \mu \mathrm{m}$ side-length squares presented in Figure S2. The deconvolution of this acquired transition with a step function reveals a measured Gaussian PSF at the object plane of $2 \sigma_{P S F} \approx 15 \mu \mathrm{m}$. By considering this PSF and the size of our object we calculate that our acquired images in Figure 3 contain approximately $\approx 17$ resolvable pixels in each transverse direction. There are many factors that limit the resolution of a trans-spectral imaging system [1,2]. However, in our ghost imaging system the resolution is primarily determined by a combination of the strength of the position correlation between signal and idler photons and the fidelity of the imaging optics

\section{Theoretical Image Resolution and Influence of Heralding Detector}

In a conventional microscope the illumination can be either spatially coherent or incoherent, albeit giving rise to subtly different point spread functions. A further subtlety in the limiting resolution of our ghost imaging system is the strength of the spatial correlations between the signal and idler photons. The degree to which the measured position of the visible signal photon recorded by the ICCD camera corresponds to that of the idler probe photon is a function of the phase-matching within the SPDC process. The standard deviation of the strength of the position correlation between signal and idler photons as measured in an image plane of the crystal is $\sigma_{x}=M \sqrt{0.455 L \lambda_{p} / 2 \pi}$ [3-5] where $M$ is the magnification between the crystal and measurement plane, $L$ is the length of the non linear crystal and a pump beam of wavelength $\lambda_{p}$, giving in the plane of our object a position correlation of $2 \sigma_{x}=5 \mu \mathrm{m}$. The resolution of our ghost imaging system cannot exceed this value, but obviously can be further degraded by the fidelity of the relay optics between the object plane and the ICCD. We note that our observed resolution is indeed lower than this predicted value a fact we attribute to the significant complexity of our image preserving delay line.
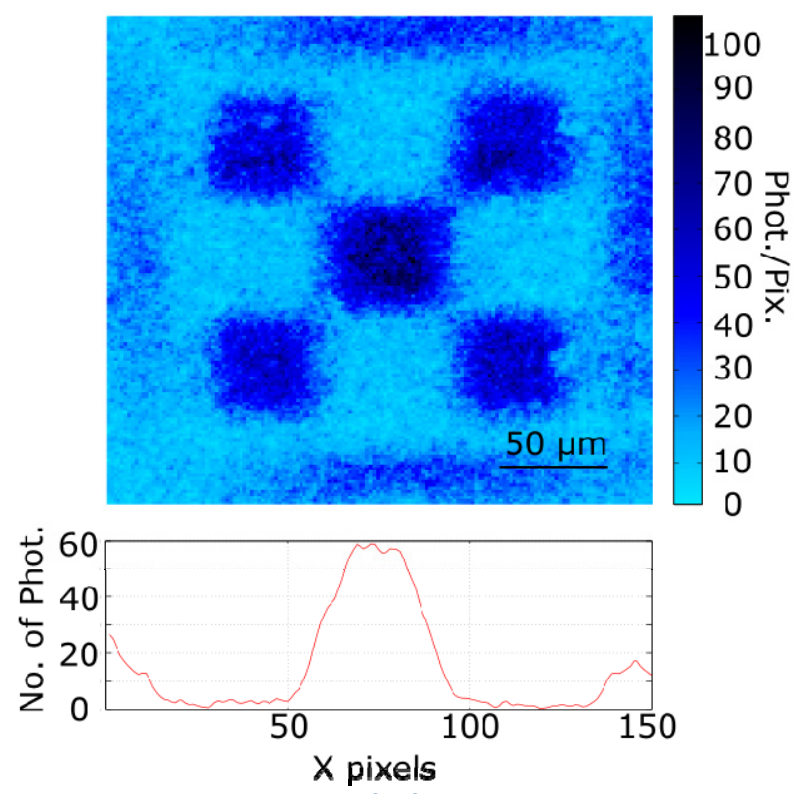

Figure S2. High contrast image of a five-spot test target. The deconvolution of the sharp transition between the edge of the gold background and silicon internal square and a step function was used to calculate the PSF, $2 \sigma P S F \approx 15 \mu \mathrm{m}$, of our system. The cross section shown is the average of the rows across the middle square of the image.

Inherent in the strength of the position correlation is the divergence of the idler field, which places a limit upon the detection efficiency of the idler photon dependent upon the numerical aperture of the relay optics between the object plane and the single-pixel heralding detector. The calculated divergence of the idler in the plane of the object corresponds to a numerical aperture (NA) of 0.1 [6]. Given the magnification of the relay optics and $0.7 \mathrm{NA}$ of the heralding detector we estimate the geometrical coupling efficiency of the idler to be of order $50 \%$. Increasing this efficiency whilst maintaining the field of view of the system would require a heralding detector of increased area and/or numerical aperture. However, we note that our current detector represents the state of the art in term of both of these parameters

\section{Image contrast}

The contrast of the acquired images is a function of both the optical efficiencies in both optical arms, and the characteristics of the source and the heralding and imaging detectors. The heralding detector has an inherent dark count rate, and whilst these do not themselves appear in the image, these dark events gate the ICCD at a random time during which there is the possibility that it detects a photon from the down-conversion source that is not correlated with any image information. Consequently, the effect of dark counts is to create a background level, mapping the emission of the source, thereby limiting the image contrast.

The rate at which image photons are acquired depends upon the product of many efficiency factors covering the optical efficiency of both the infrared (see above) and visible relay optics, the quantum efficiency of both heralding and imaging detector and the rate at which the down-converted photons are generated. However, the pair generation rate cannot be arbitrarily increased. Fundamental to the operation of our approach is that the infrared and visible photons are spatially correlated. If multiple photon pairs are generated within the gate time of the ICCD camera then the spatial correlation between two detected photons is no longer guaranteed. Under conditions of multiple photon pairs, the 
detected visible photons again map the emission profile of the source and may contain no image information. This limits the useful pair generation rate in accordance with the Poissonian statistics within the gate time of the camera. In our case we use a pair generation rate of approximately $10^{6}$ photon pairs per second. In our experiment, we used a free-space coupled InGaAs/InP SPAD as the heralding detector. This has the advantage of a relatively large detector area $(25 \mu \mathrm{m}$ diameter $)$ and high NA ( 0.7), leading to a good detection efficiency of idler probe photons. Its main disadvantages are a high dark count rate (typically $\sim 9,000 \mathrm{cps}$ at $230 \mathrm{~K}$ when the detection efficiency is set to $\sim 20 \%$ ) and a maximum trigger rate limited by a necessary hold-off time between detections to avoid after-pulsing effects (which was typically set to $10 \mu \mathrm{s}$ in these measurements, thus limiting the maximum count rate to $100 \times 10^{3}$ counts per second (cps), well above the count rate of the idler photons in this experiment). However, the gated-mode operation was exploited to effectively reduce the rate of false idler detection by using a $1.4 \mathrm{~ns}$ duration gate synchronous with the source. Additionally, sine-wave gating of similar InGaAs/InP SPAD detectors have been shown to allow detection rates in excess of $100 \times 10^{6} \mathrm{cps}$ [7]. It is possible to use a single-photon sensitive detector based on superconducting nanowires (SNSPD) as a heralding detector [8]. However, current state of the art SNSPD detectors are mainly limited to single-mode collection and thus have a low NA and small detector diameter leading to a poor detection efficiency for idler probe photons of $1 / \mathrm{N}$ where $\mathrm{N}$ is the number of resolvable image pixels. Additionally, SNSPD operates at very low temperature (less than 4 $\mathrm{K}$ ), thus requiring bulky cryogenic coolers.

However, latest generation of SNSPDs have near unity efficiency, coupled with low dark counts and deadtimes $\sim 10 \mathrm{~ns}$ [9] and as such can acquire images more rapidly and with a potentially much higher image contrast. It is anticipated that multimode SNSPD's currently under development will enable a more efficient acquisition of higher contrast images.

\section{Photon counting within each exposure of the camera}

We are able to count the number of photons in the image by applying the following methodology [10]. During each firing of the intensifier, a charge is built up on each pixel, regardless of any signal present. We measure this by acquiring 100 dark images while blocking the input to the camera. Based on a histogram of these dark signals, a threshold signal value for each pixel is determined. When in imaging mode, a pixel signal greater than this threshold value is classified as a photon detection. We apply this threshold to each acquired frame and binarise the result. The number of photons in the image is the summation of these binary signals. The short gate time (10 ns) applied to our intensifier virtually eliminates any background counts. This elimination of the background combined with single-photon nature of our imaging system allows us to acquire images using a very small number of photons, i.e. with very low photon flux.

\section{Image Reconstruction}

To reconstruct the image from the raw data we follow the technique described in [11]. In essence, we minimise the total variation-squared of our image, subject to constraints imposed by the Poissonian nature of our data. We denote the measured photon number for each of the $N$ image pixels to be $\phi_{j}$ and define the square of the total variation of the image as

$$
R\left(\zeta_{j}\right)=\sum_{j=1}^{N}\left|\frac{d \zeta_{j}}{d x}\right|^{2}+\left|\frac{d \zeta_{j}}{d y}\right|^{2}
$$

We also define a log likelihood function, L, of the reconstructed image as being

$$
\ln L=\sum_{j=1}^{N} \phi_{j} \ln \left(\zeta_{j}+\varepsilon\right)-\left(\zeta_{j}+\varepsilon\right)-\ln \left(\phi_{j} !\right)
$$

where $\zeta_{j}$ is the optimized data (as expressed in fractions of a photon) and $\mathcal{E}$ is the average dark counts for each pixel.

The optimization of the reconstructed images is based upon iterative changes to the image to increase the merit function, which combines both the fit of the image to the data and its total variation-squared.

$M=\ln L-\lambda R$

$\lambda$ is the weighting factor that sets a balance between a solution that satisfies the raw data and a solution that minimizes the total variation. Repeated iterative changes of pixel values within the image are performed and the image corresponding to a maximization of this merit function is found. If $\lambda$ is set to zero then the optimized image corresponds exactly to the data recorded, whereas if $\lambda$ is set to a very high value, the optimized image corresponds to a uniform level. In practice, the value of $\lambda$ is set empirically or statistically at an intermediate value to give a reconstructed image, see Figure S3.

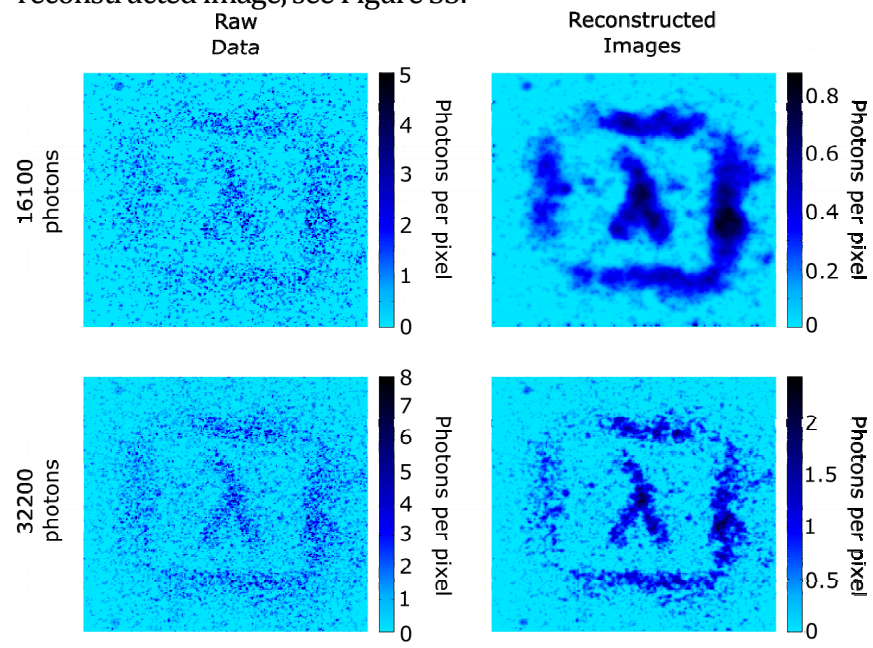

Figure S3. : Raw and reconstructed images. The images shown on the left hand side are the summation of 16100 and 32200 photons detected on the camera. We use optimisation techniques to reconstruct the images shown in the right hand column.

\section{References}

1. S. Karmakar, and Y. Shih, "Two-color ghost imaging with enhanced angular resolving power," Phys. Rev. A 81, 033845 (2010).

2. M. H. Rubin, and $Y$. Shih, "Resolution of ghost imaging for nondegenerate spontaneous parametric down-conversion," Phys. Rev. A 78, 7 (2008).

3. K. Chan, J. P. Torres, and J. H. Eberly, "Transverse entanglement migration in Hilbert space," Phys. Rev. A 75, 050101 (2007).

4. M. P. Edgar, D. S. Tasca, F. Izdebski, R. E. Warburton, J. Leach, M. Agnew, G. S. Buller, R. W. Boyd and M. J. Padgett, "Imaging highdimensional spatial entanglement with a camera," Nat. Commun. 3, 984 (2012).

5. J. Schneeloch, and J. C. Howell, "Introduction to the Transverse Spatial Correlations in Spontaneous Parametric Down-Conversion through the Biphoton Birth Zone," arXiv:1502.06996v2 [quant-ph] (2015) 
6. M. Padgett, "On the focussing of light, as limited by the uncertainty principle," J. Mod. Optic. 55, 3083 (2008).

7. C. Scarcella, G. Boso, A. Ruggeri, and A. Tosi, "InGaAs/InP Single-Photon Detector Gated at $1.3 \mathrm{GHz}$ With 1.5\% Afterpulsing," Selected Topics in Quantum Electronics, IEEE J. 21, 1 (2015).

8. C. M. Natarajan, M. G. Tanner, and R. H. Hadfield, "Superconducting nanowire single-photon detectors: physics and applications," Supercond. Sci. Technol. 25, 063001 (2012).

9. F. Marsili, V. B. Verma, J. A. Stern, S. Harrington, A. E. Lita, T. Gerrits, I. Vayshenker, B. Baek, M. D. Shaw, R. P. Mirin, and S. W. Nam. "Detecting single infrared photons with 93\% system efficiency". Nat Photonics 7, 210 (2013).

10. D. S. Tasca, M. P. Edgar, F. Izdebski, G. S. Buller, and M. J. Padgett, "Optimizing the use of detector arrays for measuring intensity correlations of photon pairs," Phys Rev A 88, 013816 (2013).

11. P. A. Morris, R. S. Aspden, J. E. C. Bell, R. W. Boyd, and M. J. Padgett, "Imaging with a small number of photons," Nat Commun 6, 5913 (2015). 